\title{
Development of a Predictive Model for Screening Patients with Psoriasis at Increased Risk of Psoriatic Arthritis
}

\author{
Yiyi Wang $\cdot$ Lingyan Zhang $\cdot$ Min Yang $\cdot$ Yanze Cao $\cdot$ Mingxin Zheng · \\ Yuanxia $\mathrm{Gu} \cdot$ Hongxiang $\mathrm{Hu} \cdot$ Hui Chen $\cdot$ Min Zhang $\cdot$ \\ Jingyi Li $\cdot$ Li Qiu $\cdot$ Wei Li (1)
}

Received: October 13, 2021 / Accepted: December 3, 2021 / Published online: December 19, 2021

(C) The Author(s) 2021

\section{ABSTRACT}

Introduction: This study aimed to develop a predictive model based on ultrasound variables which can be used to screen patients with psoriasis who are prone to progress to psoriatic arthritis (PsA) in clinical practice.

Methods: This is a cross-sectional study conducted in a single center from October 2018 to November 2020. All subjects (non-PsA group, PsA group, and control group) underwent an

Yiyi Wang and Lingyan Zhang contributed equally to this work.

Y. Wang - Y. Gu $\cdot$ H. Hu - H. Chen · M. Zhang . J. Li (ه) . W. Li (ه)

Department of Dermatology, Rare Diseases Center, West China Hospital, Sichuan University, Chengdu 610041, China

e-mail: 1jy7733@163.com

W. Li

e-mail: liweihx_hxyy@scu.edu.cn

L. Zhang · L. Qiu (ه)

Department of Ultrasound, West China Hospital, Sichuan University, Chengdu 610041, China

e-mail: wsqiuli@126.com

M. Yang

Department of Rheumatology, Rare Diseases Center, West China Hospital, Sichuan University, Chengdu 610041, China

Y. Cao $\cdot$ M. Zheng

Neusoft Corporation, Dalian 116085, China ultrasound examination and their ultrasound abnormalities were recorded. On the basis of statistical analysis and clinical experts' advice, several variables were selected for modelling. We used logistic regression to establish the prediction model. To assess the discrimination and accuracy of this model, internal validation and external validation were performed.

Results: A total of 852 patients with psoriasis but without PsA, 261 patients with PsA, and 86 healthy volunteers were included. Ultimately, the predictive model consisted of six variables, namely hand joint power Doppler (PD) signals (grade 0: OR 2.94, 95\% CI 1.94-4.47; grade $\geq 1$ : OR 109.30, 95\% CI 14.35-832.27; $P<0.001$ ), wrist joint synovial thickening (grade 1: OR 1.29, 95\% CI 0.69-2.43; grade 2: OR 4.30, 95\% CI 1.92-9.65; grade 3: OR 11.05 , 95\% CI $1.01-120.64 ; P=0.001)$, knee joint PD signals (grade 0: OR 1.01, 95\% CI 0.56-1.80; grade $\geq 1$ : OR 14.77, 95\% CI 3.99-54.69; $P<0.001)$, toe joint PD signals (grade 0: OR 1.18, 95\% CI $0.78-1.79 ; \quad$ grade $\geq 1$ : OR 5.74, $95 \% \mathrm{CI}$ $2.84-11.63 ; P<0.001)$, quadriceps tendon and patellar tendon enthesitis (OR 1.95, 95\% CI 1.36-2.78, $P<0.001)$, Achilles tendon and plantar aponeurosis enthesitis (OR 1.63, 95\% CI $1.14-2.32, P=0.007)$. C-index for the predictive model was 0.80 (95\% CI 0.76-0.83). After bootstrapping validation (1000 times), it was confirmed to be 0.79 . The external validation showed the accuracy of the predictive model is 0.87 (95\% CI 0.69-0.95). 
Conclusion: This study succeeded in developing a predictive model with a high degree of accuracy to predict the risk of PsA in patients with psoriasis.

\section{PLAIN LANGUAGE SUMMARY}

Psoriatic arthritis often occurs in the population of patients with psoriasis. It brings a huge burden and pain to patients. At present, the diagnosis for psoriatic arthritis is very challenging. Numerous research studies have begun to focus on identifying patients with psoriasis at increased risk of psoriatic arthritis. Among a lot of modalities, ultrasound has been considered as a sensitive and convenient tool for screening early psoriatic arthritis. Our study successfully established a predictive model based on ultrasound variables to screen patients with psoriasis at high risk of transiting to psoriatic arthritis. After internal and external validation, it showed great accuracy and generalizability. We recommend that clinicians perform ultrasound screening of patients with psoriasis in clinical routine and get their risk value of transiting to psoriatic arthritis by using this model. For those patients with a high risk of progression to psoriatic arthritis, clinicians should refer them to a rheumatology department as soon as possible so that they could have access to early and effective management which might bring them good clinical and imaging outcomes.

Keywords: Psoriasis; Psoriatic arthritis; Ultrasound; Predictive model

\section{Key Summary Points}

Why carry out this study?

At present, for psoriatic arthritis, the most important thing is to screen the patients with psoriasis at high risk of psoriatic arthritis transition who might benefit from early intervention that could improve clinical and imaging outcomes
This study aimed to develop a predictive model based on ultrasound variables which can be used to screen patients with psoriasis who are prone to progress to psoriatic arthritis in clinical practice

\section{What was learned from the study?}

Our study provides clinicians with an effective and simple predictive nomogram for the early screening of patients with psoriasis at high risk of transiting to psoriatic arthritis

This predictive model is recommended for dermatologists in their daily clinical work to screen for early psoriatic arthritis

\section{INTRODUCTION}

Psoriatic arthritis (PsA) is a chronic inflammatory musculoskeletal disease with an incidence of $6-41 \%$ in the psoriatic population [1], typically presenting as synovitis, enthesitis, dactylitis, and sacroiliitis [2]. PsA can even develop into destructive arthritis in some patients [2] and patients with PsA have lower quality of life and life expectancy [3]. Because of heterogeneity and complicacy, PsA remains very challenging in terms of diagnosis [2]. Despite the wide use of classification Criteria for Psoriatic Arthritis (CASPAR) in both clinical research and clinical practice, patients who meet the criteria have presented the inflammatory musculoskeletal disease [4]. However, any treatment might not improve the longterm clinical outcomes for patients with psoriasis who have clinically confirmed arthritis [5]. Therefore, at present, the most important thing is to identify the patients with psoriasis at high risk of PsA transition who might benefit from early intervention that could improve clinical and imaging outcomes [5]. As most patients with PsA have psoriatic skin lesions prior to arthropathy, the cutaneous biomarker is therefore suitable for dermatologists to screen and recognize early PsA [6]. 
There are various modalities to screen for early PsA. Among them, ultrasound (US) is a relatively more sensitive and convenient modality for assessing not only the disease activity but also damage of inflammatory arthritis compared with physical examination and other imaging methods [7-11]. More and more studies have noted that US could detect subclinical imaging changes in patients with psoriasis without musculoskeletal symptoms [12-14]. In addition, a few studies showed that enthesopathy and hand synovitis detected by US or magnetic resonance imaging could predict the development of PsA $[15,16]$. However, despite US being able to sensitively detect a number of abnormalities in patients with early PsA, faced with a mass of ultrasound variables, it is difficult for clinicians to determine which of those changes could better predict the progression of PsA in patients with psoriasis. Therefore, it is essential to establish a quantified, visual, and convenient tool based on ultrasound variables to predict risk of future clinically evident PsA for patients with psoriasis.

In this study, we aim to establish a predictive model based on ultrasonic variables which can be used to identify patients with psoriasis at increased risk of PsA in clinical practice.

\section{METHODS}

\section{Study Design and Patients}

This cross-sectional study comprised patients with psoriasis without clinical symptoms and signs of psoriatic arthritis (non-PsA group), patients with psoriatic arthritis (PsA group), and healthy volunteers (control group). All patients with psoriasis came from the Department of Dermatology, West China Hospital, Sichuan University between October 2018 and November 2020. Healthy volunteers came from the physical examination center at West China Hospital, Sichuan University. All subjects underwent elaborate ultrasound examination. The study was approved by the ethics committee of West China Hospital, Sichuan University (approval number ChiCTR-DCD-15006851) and performed in accordance with the Helsinki
Declaration of 1964, and its later amendments. Each participating patient signed an informed consent form. No identifying information of participants was included in the manuscript.

\section{Inclusion and Exclusion Criteria}

In accordance with the inclusion and exclusion criteria of clinical studies in our center [17], the inclusion criteria for the non-PsA group were as follows: (i) aged 18-65 years with no gender restriction; (ii) with a clear diagnosis of psoriasis confirmed by two experienced dermatologists. Exclusion criteria were (i) current or historical diagnosis of any arthritis including psoriatic arthritis, rheumatoid arthritis, osteoarthritis, gouty arthritis, ankylosing spondylitis, and similar conditions; (ii) pregnancy; (iii) a history of trauma or heavy manual labor prior to US examination; (iv) with other comorbidities including hematological system diseases, malignant tumors, serious hepatic dysfunction, renal dysfunction, or other visceral organ dysfunction.

For the PsA group, patients included should meet the following inclusion criteria: (i) aged 18-65 years, irrespective of gender; (ii) met the Classification Criteria for Psoriasis Arthritis [4] according to which patients must have a precondition of inflammatory musculoskeletal disease and meanwhile achieve a score $\geq 3$ among the following five categories: evidence of psoriasis (current psoriasis, a personal history of psoriasis, or a family history of psoriasis), presence of psoriatic nail dystrophy, a negative test for rheumatoid factor, dactylitis (current or history), and radiographic evidence of juxta articular new bone formation. Patients were excluded if they met the following exclusion criteria: (i) a diagnosis of any other arthritis including rheumatoid arthritis, osteoarthritis, gouty arthritis, ankylosing spondylitis, and similar conditions; (ii) pregnancy; (iii) a history of trauma or heavy manual labor prior to US examination; (iv) with other comorbidities including hematological system diseases, malignant tumors, serious hepatic dysfunction, renal dysfunction, or other visceral organ dysfunction. 
Finally, we selected healthy volunteers as the control group. For the control group, exclusion criteria were as follows: (i) pregnancy; (ii) a history of trauma or heavy manual labor prior to US examination; and (iii) a current or previous diagnosis of any diseases including psoriasis and any other skin diseases, psoriatic arthritis and any other form of arthritis, hematological system diseases, malignant tumors, serious visceral organ dysfunction, and similar conditions.

\section{US Examination}

The ultrasound examinations were performed by three sonographers with more than 5 years of experience in musculoskeletal ultrasound imaging. To ensure that the evaluation for each subject was homogeneous, all operating procedures referred to the guideline for musculoskeletal ultrasound examination jointly developed by the American Society of Ultrasound in Medicine (AIUM) [18]. All results were checked by an experienced sonographer (LZ) to guarantee the correctness of the results. The examination time was approximately $30 \mathrm{~min}$ per subject. The radiologists were blinded to subjects' clinical data. In addition, all subjects were required not to communicate about their diseases with the sonographer during the US examination.

The device used was a color power Doppler (PD) US (Philips IU22). Depending on the depth of joint, the probe frequency was set at 3-9 or $5-12 \mathrm{MHz}$, and the musculoskeletal condition was chosen. The sonographer examined all target joints, tendons, bursae, and nails in grayscale mode and detected the blood flow signals in PD mode. The grayscale pattern showed structural changes and the gain was set at maximum sensitivity without noise signals. The PD pattern showed the activity level of inflammation and its gain was set at maximum sensitivity without Doppler artifact and color overflow.

The joints scanned included shoulders, elbows, wrists, metacarpophalangeal joints (MCP), interphalangeal joints (IP) of the fingers, hips, knees, ankles, tarsal joints, metatarsophalangeal joints (MTP), and interphalangeal joints of the toes. All recesses in the above articular cavity were completely scanned. Among them, wrists, metacarpophalangeal joints, metatarsophalangeal joints, and interphalangeal joints were both dorsally and palmarly scanned. The tendons scanned included long heads of the biceps brachii, supraspinatus tendon, infraspinatus tendon, teres minor tendon, common extensor tendon, common flexor tendon, triceps tendon, extensor and flexor tendons of wrists and hands, gluteus medius tendon, gluteus minor tendon, iliotibial band, quadriceps tendon, tibialis anterior tendon, tibial posterior tendons, peroneal long/short tendons, Achilles tendons, plantar fascia, flexor and extensor tendons of the toes. The bursae scanned included subacromial-subdeltoid bursae, olecranon bursae, gastrocnemiussemimembranosus bursae, and posterior calcaneal bursae. All tendons and bursae underwent transverse and longitudinal scanning. The nails scanned included the superficial and deep nail plate, nail bed, and nail matrix of each fingernail and toenail.

For joints, joint effusion, synovial thickening, PD signals, osteophytes, and bone erosions were evaluated. Similarly, for tendons, enthesitis (entheses thickening, hypoechogenicity, PD signals, osteophytes, calcifications, and bone erosions), tendon sheath synovial thickening, and tendon sheath effusion were evaluated. With respect to bursae, the sonographer evaluated bursa synovial thickening and bursa effusion. Regrading nail dystrophy, the sonographer mainly observed whether there was thickening of the nail plate structure, surface unevenness, and disappearance of the deep nail plate and whether the echogenicity and blood flow signal of nail bed and nail matrix were altered.

Among these variables, joint effusion, joint synovial thickening, and joint PD signals were semiquantitatively assessed. The joint effusion and joint synovial thickening were scored as follows [19]: grade 0 (no anechoic, hypoechoic, or hyperechoic structure was visible, which means no effusion/synovial thickening), grade 1 (joint effusion/synovial thickening did not exceed the peripheral trigonum of the bones adjacent to the scanned joint), grade 2 (joint effusion/synovial thickening extended 
beyond the articular cavity but did not reach the backbone), grade 3 (joint effusion/synovial thickening extended beyond the articular cavity and reached the backbone). The grade of unilateral multi-joints (such as MCP, MTP, and IP) were recorded with the highest score. Finally, for joints with synovial thickening, the joint PD signals were scored following the following criteria [20]: grade 0 (no intra-articular blood flow signals), grade 1 (no more than three blood flow signals), grade 2 (a small amount of blood flow signals, the area was less than $50 \%$ of the synovial area), grade 3 (continuous blood flow signals, the area exceeded $50 \%$ of the synovial area). Likewise, the grades of unilateral multijoints (such as MCP, MTP, and IP) were recorded with the highest score.

\section{Statistical Analysis}

Categorical variables were expressed as count (\%), while continuous variables were represented using mean (SD). The alpha level was set to 0.05 with all tests two-sided. A $P$ value less than 0.05 was statistically significant. We used Fisher's exact test to compare the categorical variables of the PsA, non-PsA, and control groups, while Student's $t$ test was used to compare the continuous variables among the three groups. Next, we went through the steps of variable selection, model establishment, and accuracy assessment to develop a prediction model. Firstly, we performed the statistical analysis of differences in all ultrasound variables between the PsA and non-PsA groups.
Then, on the basis of the opinions of clinical experts, the most clinically significant variables were selected. Logistic regression was used as our modeling method to classify the two groups (PsA and non-PsA), and we used a nomogram to show this prediction model, which is a practical tool to visualize the results. C-index, a generalization of the area under the receiver operating characteristic (ROC), was used to quantify the performance of this prediction model, and the established nomogram was evaluated by drawing the calibration curve. To avoid worse clinical outcomes, decision curve analysis (DCA) was performed to evaluate the clinical usefulness of the prediction model by calculating the net income under different threshold probabilities between the two groups. Statistical analysis was performed with RStudio software (Version 4.0.2, https://www.R-project.org).

\section{RESULTS}

\section{Patient Characteristics}

A total of 852 patients in the non-PsA group, 261 patients with PsA, and 86 healthy volunteers were included in the study. The demographics of these subjects are shown in Table 1. Gender, height, weight, and body mass index (BMI) among the three groups showed no significant difference (all $P>0.05$ ).

Table 1 Demographics of the non-PsA, PsA, and control groups

\begin{tabular}{lcccc}
\hline Variables & Non-PsA $(\boldsymbol{n}=\mathbf{8 5 2})$ & PsA $(\boldsymbol{n}=\mathbf{2 6 1})$ & Control $(\boldsymbol{n}=\mathbf{8 6})$ & $\boldsymbol{P}$ value \\
\hline Age, years & $39.53(14.65)$ & $41.20(10.11)$ & $40.48(11.94)$ & 0.21 \\
Gender, male, $n(\%)$ & $549(64.44)$ & $163(62.45)$ & $59(68.60)$ & 0.58 \\
Height, cm & $165.54(8.06)$ & $164.11(8.05)$ & $164.83(8.27)$ & 0.21 \\
Weight, cm & $65.45(13.09)$ & $63.99(11.37)$ & $64.02(7.84)$ & 0.38 \\
BMI, $\mathrm{kg} / \mathrm{m}^{2}$ & $23.73(3.79)$ & $23.74(3.73)$ & $23.50(1.52)$ & 0.84 \\
\hline
\end{tabular}

Data are presented as mean (SD) unless otherwise specified

$B M I$ body mass index 


\section{Most Important Affected Anatomical Sites of PsA}

The top ten most common affected anatomical sites of PsA are quadriceps tendon, MTP, Achilles tendon, proximal interphalangeal joint (PIP), knee, MCP, ankle, wrist, distal interphalangeal joint (DIP), and shoulder with the prevalence of $55.17 \%, 54.02 \%, 50.57 \%, 47.89 \%$, $46.74 \%, 39.85 \%, 32.57 \%, 27.20 \%, 24.14 \%$, and $20.69 \%$, respectively (Fig. 1 ).

\section{Comparison of Ultrasound Abnormalities Between the Three Groups}

In joint changes, the percentage of joint effusion, synovial thickening, PD signals, osteophytes, and bone erosion of patients with PsA was significantly higher than that of patients in the non-PsA group (all $P<0.05$ ). Entheses thickening, hypoechogenicity, PD signals, osteophytes, and bone erosion in patients with PsA were significantly higher than in patients in the non-PsA group (all $P<0.05$ ). With respect to tendon changes, patients with PsA presented more tendon sheath synovial thickening and tendon sheath effusion than patients in the non-PsA group (all $P<0.05$ ). Bursa synovial thickening also occurred more frequently in the PsA group than in the non-PsA group $(P<0.05)$. Moreover, compared with healthy controls, patients in the non-PsA group were found to have several subclinical ultrasonic abnormalities including joint effusion, joint synovial thickening, joint PD signals, joint osteophytes, entheses hypoechogenicity, and tendon sheath effusion (all $P<0.05$ ). The details are summarized in Table 2 .

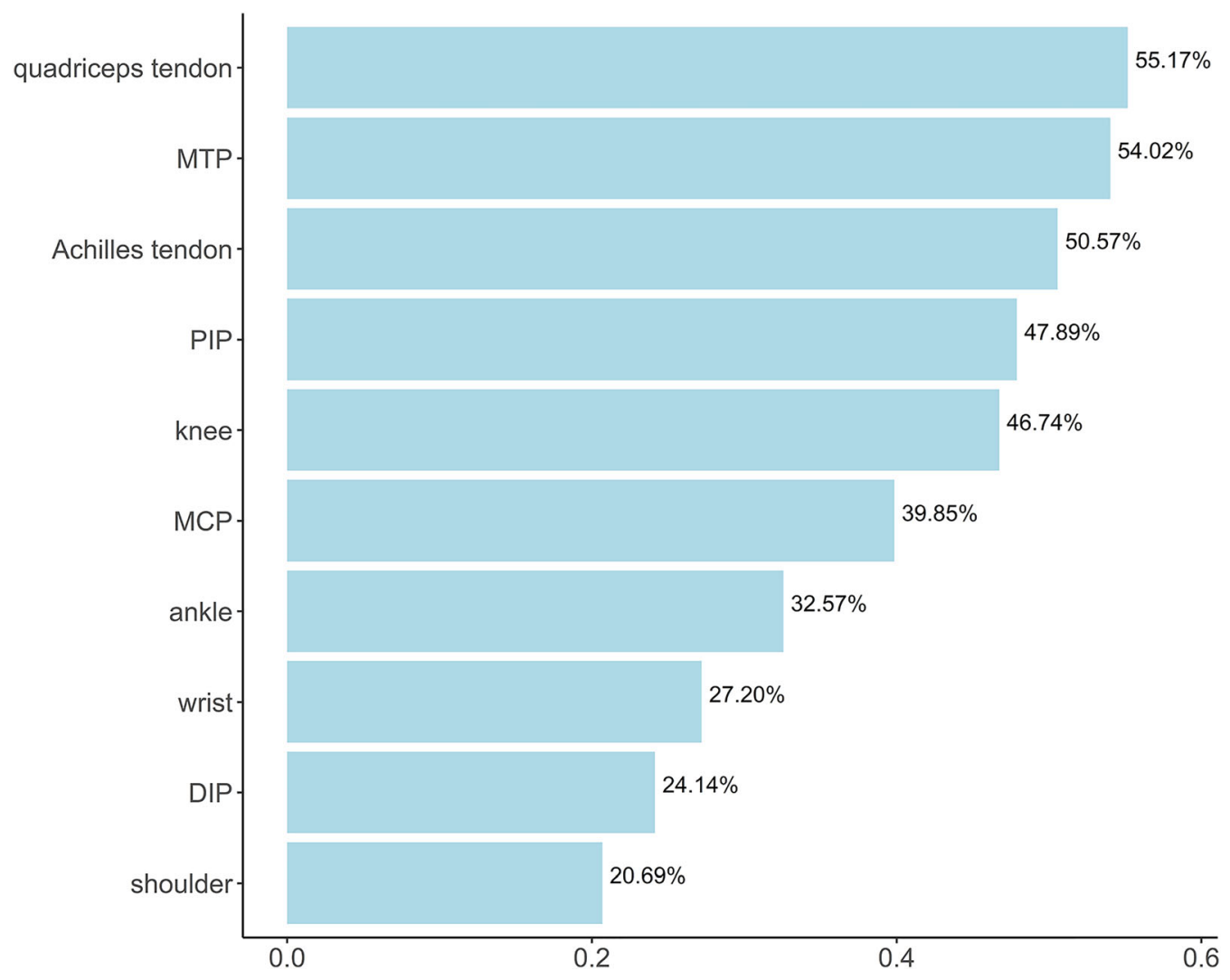

Fig. 1 Top10 most common affected anatomical sites of PsA. MTP metatarsophalangeal joints, PIP proximal interphalangeal joints, $M C P$ metacarpophalangeal joints, DIP distal interphalangeal joint 
Table 2 Differences of ultrasound changes among the non-PsA, PsA, and control groups

\begin{tabular}{|c|c|c|c|c|c|}
\hline Features & Non-PsA $(n=852)$ & PsA $(n=261)$ & Control $(n=86)$ & $P$ value* & $P$ value $\#$ \\
\hline \multicolumn{6}{|l|}{ Joint changes } \\
\hline Joint effusion & $410(0.48)$ & $154(0.59)$ & $22(0.26)$ & 0.003 & $<0.001$ \\
\hline Joint synovial thickening & $277(0.33)$ & $186(0.71)$ & $9(0.10)$ & $<0.001$ & $<0.001$ \\
\hline Joint PD signals & $273(0.32)$ & $185(0.71)$ & $9(0.10)$ & $<0.001$ & $<0.001$ \\
\hline Joint osteophytes & $119(0.14)$ & $104(0.40)$ & $3(0.03)$ & $<0.001$ & 0.01 \\
\hline Joint bone erosion & $16(0.02)$ & $76(0.29)$ & $0(0.00)$ & $<0.001$ & 0.40 \\
\hline \multicolumn{6}{|l|}{ Entheses changes } \\
\hline Entheses thickening & $281(0.33)$ & $158(0.61)$ & $26(0.30)$ & $<0.001$ & 0.69 \\
\hline Entheses hypoechogenicity & $82(0.10)$ & $75(0.29)$ & $1(0.01)$ & $<0.001$ & 0.02 \\
\hline Entheses PD signals & $32(0.04)$ & $38(0.15)$ & $0(0.00)$ & $<0.001$ & 0.13 \\
\hline Entheses osteophytes & $371(0.44)$ & $183(0.70)$ & $38(0.44)$ & $<0.001$ & 1.00 \\
\hline Entheses calcifications & $34(0.04)$ & $16(0.06)$ & $4(0.05)$ & 0.20 & 0.99 \\
\hline Entheses bone erosion & $12(0.01)$ & $24(0.09)$ & $0(0.00)$ & $<0.001$ & 0.55 \\
\hline \multicolumn{6}{|l|}{ Tendon changes } \\
\hline Tendon sheath synovial thickening & $27(0.03)$ & $81(0.31)$ & $1(0.01)$ & $<0.001$ & 0.48 \\
\hline Tendon sheath effusion & $71(0.08)$ & $36(0.14)$ & $0(0.00)$ & 0.01 & 0.01 \\
\hline \multicolumn{6}{|l|}{ Bursa changes } \\
\hline Bursa synovial thickening & $32(0.04)$ & $53(0.20)$ & $5(0.06)$ & $<0.001$ & 0.52 \\
\hline Bursa effusion & $33(0.04)$ & $16(0.06)$ & $0(0.00)$ & 0.17 & 0.12 \\
\hline Nail dystrophy & $263(30.87)$ & $97(37.16)$ & $0(0.00)$ & 0.06 & $<0.001$ \\
\hline
\end{tabular}

Data are presented as $n(\%)$

$P D$ power Doppler

${ }^{*} P$ value between the non-PsA and PsA groups

${ }^{\#} P$ value between the non-PsA and control groups

\section{Development of Predictive Model}

According to the suggestions of clinical experts, we selected the following variables: synovial thickening and PD signals of knee joints, ankle joints, wrist joints, hand joints (MCP and IP), and toe joints (MTP and IP), quadriceps tendon and patellar tendon enthesitis, Achilles tendon and plantar aponeurosis enthesitis for variable selection. On the basis of statistical analysis using the forward:LR selection method, we identified six variables (hand joint PD signals, wrist joint synovial thickening, knee joint PD signals, toe joint PD signals, quadriceps tendon and patellar tendon enthesitis, Achilles tendon and plantar aponeurosis enthesitis) that could make significant contributions to the risk prediction model (with $P<0.05$ ), in which the other six variables were excluded because their $P$ values were greater than 0.05 (Table 3 ). There are some steps that should be followed when using this nomogram (Fig. 2):

1. Enthesitis-related variables were rated as yes and no. 
Table 3 Logistic regression model for PsA risk prediction

\begin{tabular}{|c|c|c|}
\hline Variables & Odds ratio $(95 \% \mathrm{CI})$ & $P$ value \\
\hline \multicolumn{3}{|l|}{ Hand joint PD signals (MCP and IP) } \\
\hline Grade 0 & $2.94(1.94-4.47)$ & $<0.001$ \\
\hline Grade $\geq 1$ & $109.30(14.35-832.27)$ & \\
\hline \multicolumn{3}{|l|}{ Wrist joint synovial thickening } \\
\hline Grade 1 & $1.29(0.69-2.43)$ & 0.001 \\
\hline Grade 2 & $4.30(1.92-9.65)$ & \\
\hline Grade 3 & $11.05(1.01-120.64)$ & \\
\hline \multicolumn{3}{|l|}{ Knee joint PD signals } \\
\hline Grade 0 & $1.01(0.56-1.80)$ & $<0.001$ \\
\hline Grade $\geq 1$ & $14.77(3.99-54.69)$ & \\
\hline \multicolumn{3}{|l|}{ Toe joint PD signals (MTP and IP) } \\
\hline Grade 0 & $1.18(0.78-1.79)$ & $<0.001$ \\
\hline Grade $\geq 1$ & $5.74(2.84-11.63)$ & \\
\hline Quadriceps tendon and patellar tendon enthesitis & $1.95(1.36-2.78)$ & $<0.001$ \\
\hline Achilles tendon and plantar aponeurosis enthesitis & $1.63(1.14-2.32)$ & 0.007 \\
\hline
\end{tabular}

$C I$ Confidence interval, $P D$ power Doppler, $M C P$ metacarpophalangeal joints, $I P$ interphalangeal joints, $M T P$ metatarsophalangeal joints

Points
quadriceps tendon and
patellar tendon
enthesitis
toes joint PD signals
achilles tendon and
plantar aponeurosis
enthesitis
hand joint PD signals
knee joint PD signals
wrist joint synovial
thickening
Total Points
Risk of PsA

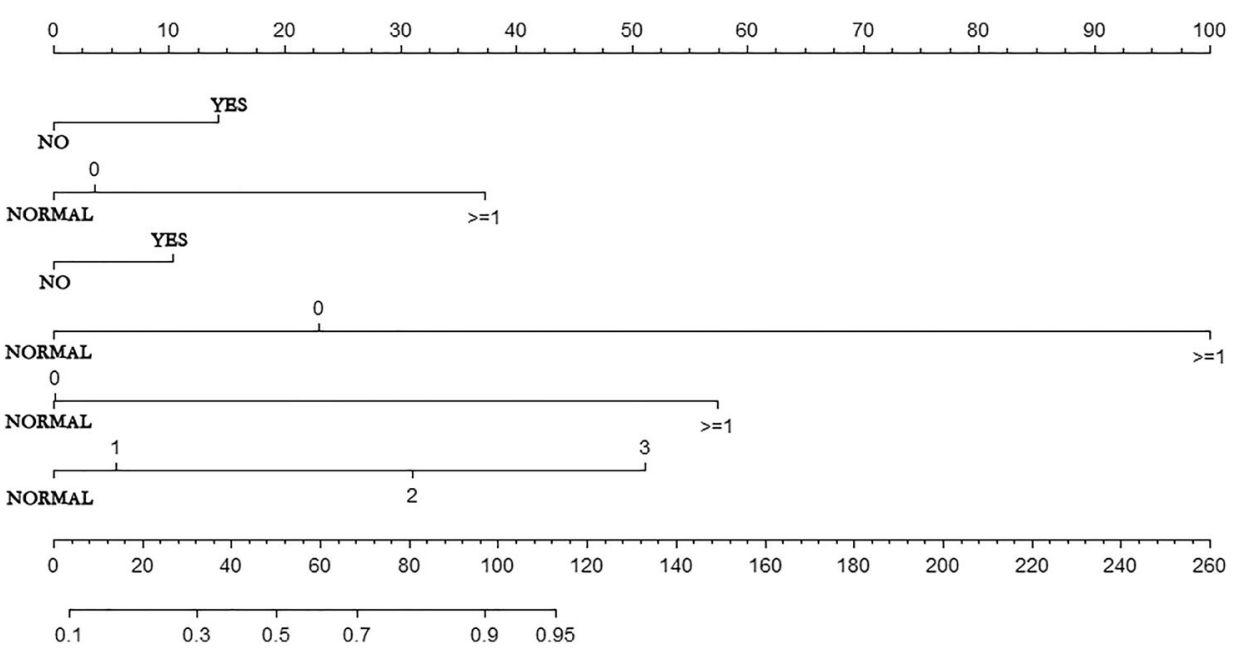

Fig. 2 Nomogram of PsA risk predictive model. PD power Doppler 
2. PD signals were rated as normal, 0 and greater than or equal to 1 , while synovial thickening was rated as normal, 1, 2, and 3 . The grading standard refers to the above methodology.

3. Each feature should be matched with its point.

4. The point of each feature should be summed up and then the total points are calculated.

5. Finally, the total points should be matched with the risk of PsA.

\section{Internal Validation for Prediction Model}

To ensure the good discrimination and accuracy of this model, internal validation was applied. The calibration curve (Fig. 3) was employed to assess the predictive power of selected variables and the scoring system. C-index for the predictive model was 0.80 (95\% CI $0.76-0.83$ ). After bootstrapping validation (1000 times), the C-index was confirmed to be 0.79 . According to receiver operating characteristic curve (ROC curve) (Fig. 4), it can also be proved that our model has a good prediction effect. The decision curve analysis (Fig. 5) for the PsA risk nomogram showed that if the threshold probability is between $1 \%$ and $93 \%$, the PsA risk nomogram is more beneficial.

\section{External Validation for Prediction Model}

To demonstrate the predictive value and generalizability of the predictive model, we performed external validation. In accordance with the inclusion and exclusion criteria of this study, we included 126 patients in the non-PsA group and 39 patients with PsA who came from the Department of Dermatology, West China Hospital, Sichuan University between December 2020 and April 2021 and performed the same ultrasound examination for them. As a result, the external validation showed the C-index for the predictive model was 0.87 (95\% CI 0.69-0.95) (Fig. 6). This demonstrated that the predictive model had a relatively high accuracy and generalizability.

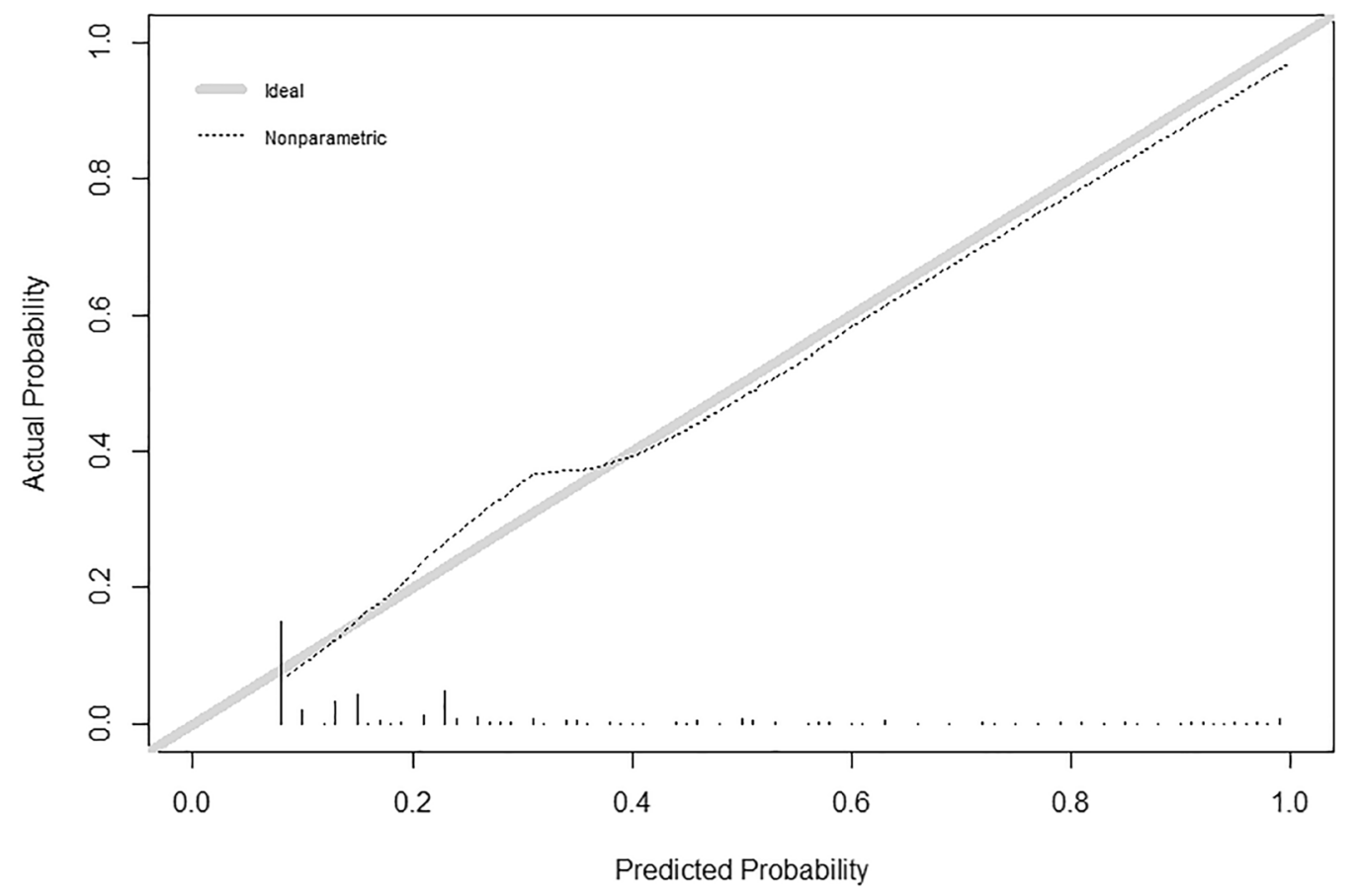

Fig. 3 Calibration curve 


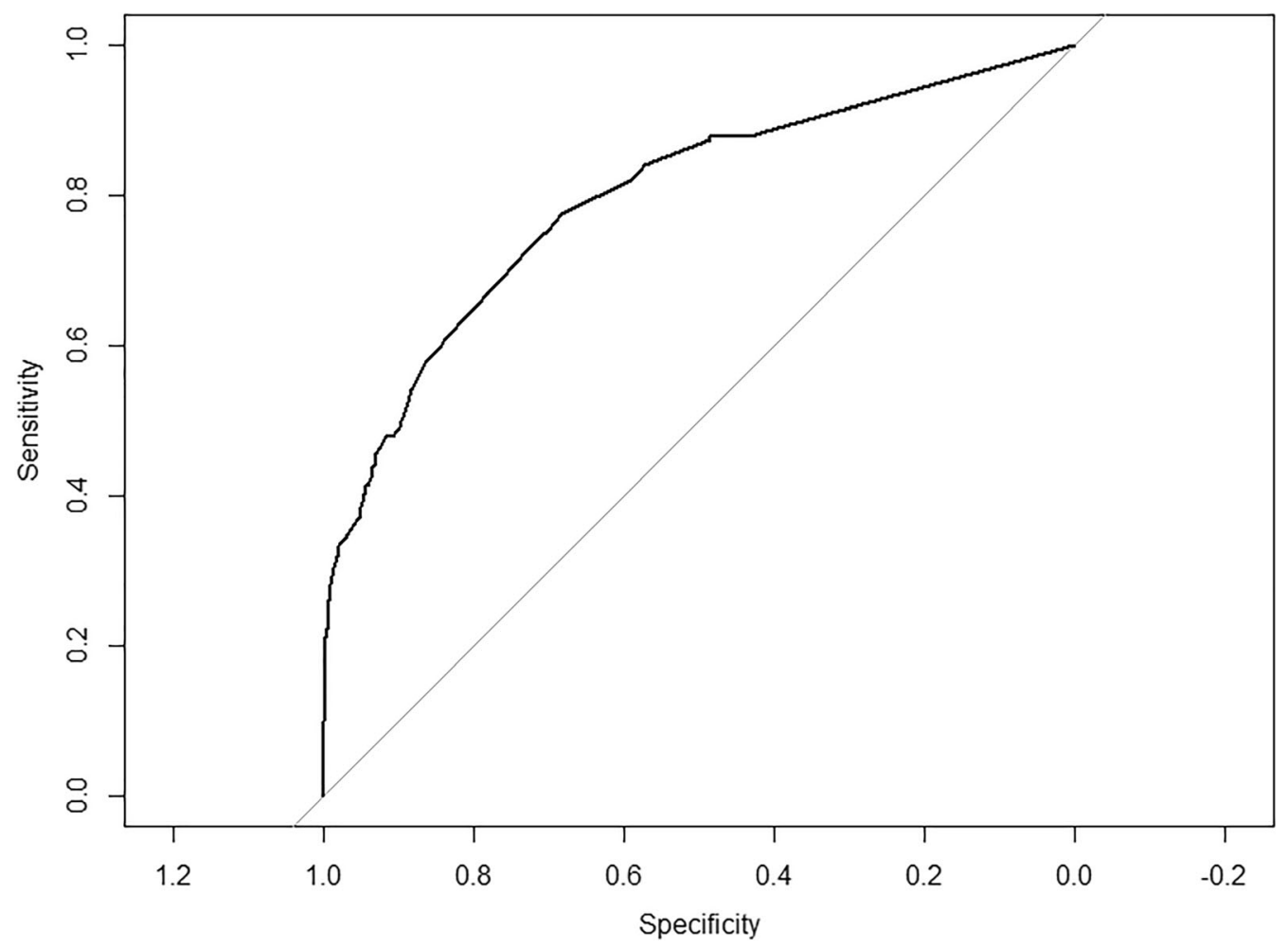

Fig. 4 Receiver operating characteristic curve

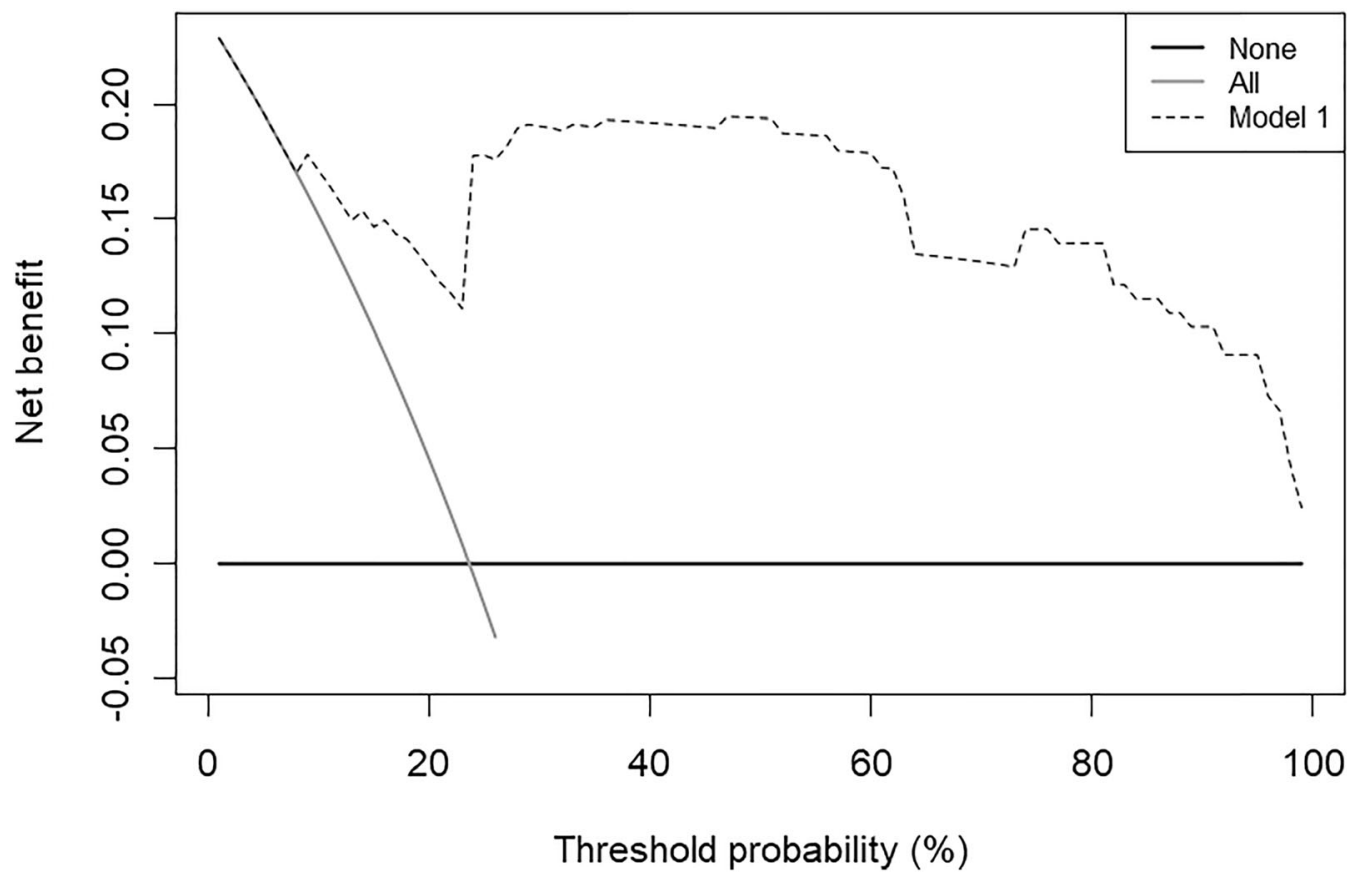

Fig. 5 Decision curve 


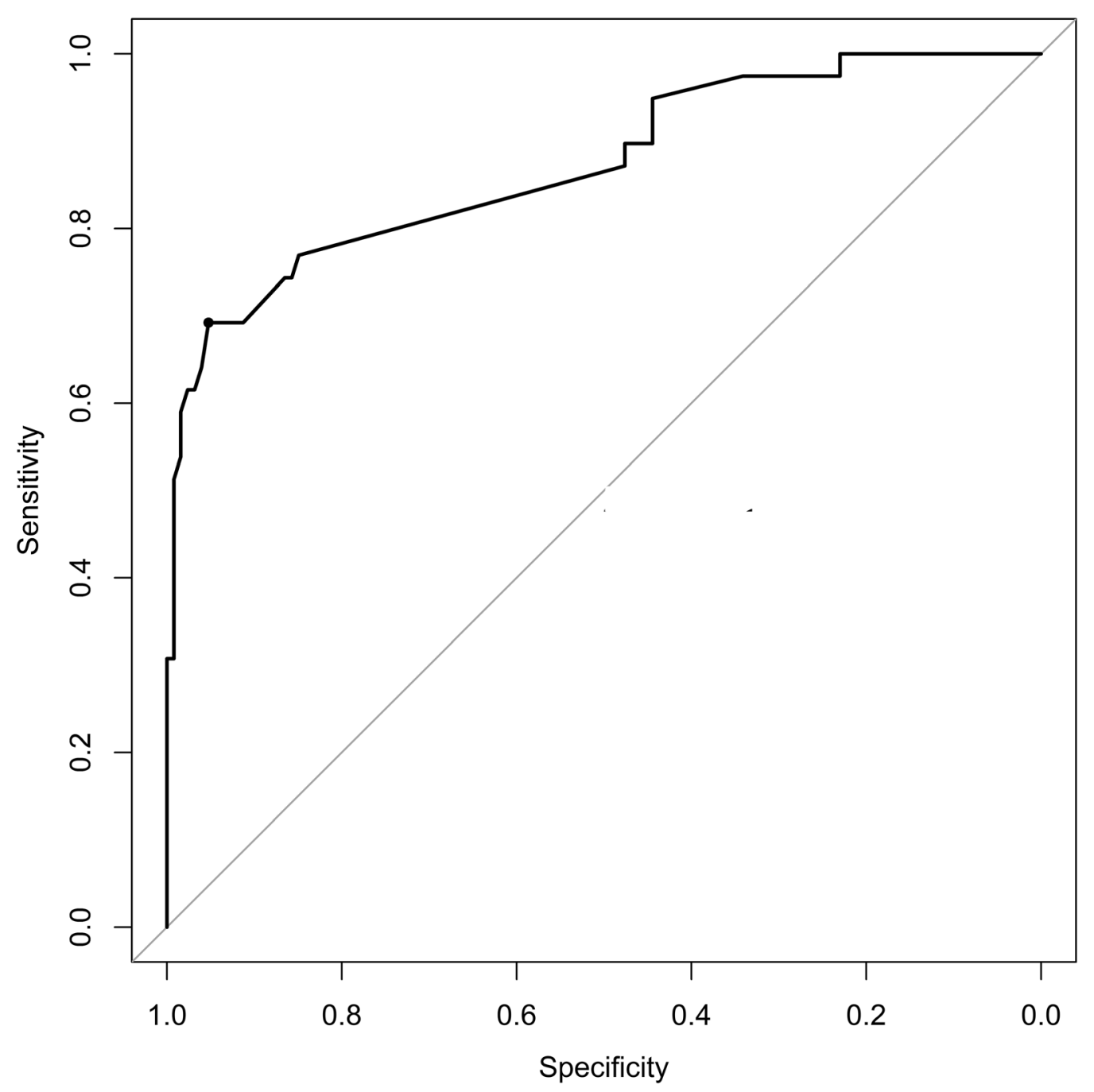

Fig. 6 Receiver operating characteristic curve for the external validation set

\section{DISCUSSION}

In this study, we successfully established a quantitative predictive model comprising six variables, namely hand joint PD signals (MCP and IP), wrist joint synovial thickening, knee joint PD signals, toe joint PD signals (MTP and IP), quadriceps tendon and patellar tendon enthesitis, Achilles tendon and plantar aponeurosis enthesitis. After internal and external validation, the model showed relatively high predictive value.

Among all the variables included in the model, hand joint PD signals, which are indicative of active inflammation [21], had the greatest contribution to the model. Consistent with this conclusion, a few previous studies showed that the inflammation at metacarpophalangeal joints and proximal interphalangeal joints could be commonly detected in early PsA by ultrasound [22]; another research study concluded that patients with psoriasis in whom hand synovitis was detected had a 55.5\% probability of developing PsA within 1 year [16]. And there is also one study suggesting that ultrasonic examination of hand joints could assist clinicians in diagnosing early PsA [23]. Taken together these findings demonstrate that the inflammation of hand joints is extremely important for the risk prediction of PsA 
progression. This conclusion is consistent with our clinical observation that patients with PsA often have disease involving the hand joints.

Furthermore, wrist joint synovial thickening, knee joint PD signals, toe joint PD signals, quadriceps tendon and patellar tendon enthesitis, Achilles tendon and plantar aponeurosis enthesitis also played significant roles in the model, suggesting that patients with these changes are more likely to develop PsA. This conclusion is also supported by numerous previous studies showing that subclinical synovitis and enthesitis could predict PsA development [24-27]. However, none of the previous studies have given specific screening sites for reference. Our results, locating the screening sites on MCP and IP of hands, wrist, knee, MTP and IP of toes, quadriceps tendon, patellar tendon, Achilles tendon, and plantar aponeurosis, could be helpful for clinicians in their busy daily work to conduct simple and effective screening.

Moreover, in this study, we also concluded that the most common affected anatomical sites of patients with PsA were quadriceps tendon, MTP, Achilles tendon, PIP, and knee, which is similar to the findings of most previous studies $[28,29]$. When conducting clinical and imaging examinations for patients with psoriasis, clinicians should pay more attention to these anatomical sites. Additionally, in patients with psoriasis without clinically confirmed arthritis, several subclinical ultrasound abnormalities including joint effusion, joint synovial thickening, joint PD signals, joint osteophytes, entheses hypoechogenicity, and tendon sheath effusion were detected, which is consistent with a number of previous studies showing that subclinical synovitis and enthesopathy exist in patients with psoriasis with no musculoskeletal symptoms and signs [21, 30-33]. In addition, in this study, we found no statistically significant difference between the PsA and non-PsA groups for nail dystrophy. In the past, several studies suggested that nail dystrophy was associated with a higher likelihood of PsA [34, 35]. However, Eder and his colleagues [36] found that only nail pitting was a strong predictor of PsA progression, while nail onycholysis was not associated with the development of PsA. This suggests that using only nail dystrophy to predict the development of PsA is inadequate, and further research in the future is needed to investigate which subtype of nail dystrophy could effectively predict the progression of PsA.

To the best of our knowledge, this is the first predictive model based on ultrasound variables to identify patients with psoriasis at increased risk of transition to PsA, which has the advantages of quantification, simplicity, and accuracy and is suitable for application in clinical practice. The fact that psoriatic skin lesion is considered as the most significant biomarker of PsA places dermatologists at an ideal juncture for the early recognition of PsA [37]. Therefore, we recommend that dermatologists perform ultrasound screening of patients with psoriasis in clinical routine, focus on the variables included in our model, score against the steps for usage of the nomogram as described above, and finally get the risk value of transiting to PsA for patients with psoriasis. For those patients with a high risk of progression to PsA, dermatologists should refer them to rheumatology department as soon as possible so that they could have access to early and effective management which might bring them good clinical and imaging outcomes $[5,38]$.

Inevitably, this study has a few limitations. First, it is a cross-sectional study with inherent defects that we can only infer correlation rather than causality. Second, a few abnormalities which might be detected in patients with psoriasis, such as peritendinous edema and dactylitis were not assessed in this study. Moreover, because axial joints including those in back and neck areas cannot be properly imaged by ultrasound, they were not included in this study. In the future, it is necessary to explore the axial abnormalities of patients with PsA by computerized tomography and/or magnetic resonance imaging. Third, since the primary aim of this study was to develop a predictive model based on ultrasound variables without incorporating other laboratory parameters or clinical manifestations, we cannot draw a definitive conclusion about which psoriasis patient population, with what specific clinical characteristics, requires further ultrasound screening and would benefit from using this predictive model. This question needs to be 
explored and validated with another study in the future. Finally, this model requires multicenter data for further external validation.

\section{CONCLUSIONS}

Our study provides clinicians with an effective and simple tool for the early screening of patients with psoriasis at high risk of transiting to PsA who could get benefit from early referral and intervention. The model still needs to be validated and optimized with more cohorts in the future.

\section{ACKNOWLEDGEMENTS}

Funding. This study including the Rapid Service Fee was funded by the 135 Project for Disciplines of Excellence, West China Hospital, Sichuan University, China (ZYJC18003) and West China Precision Medicine Industrial Technology Institutes (2018-CY02-00058-GX) and Sichuan Provincial Science and Technology Project (2021YFG0306).

Medical Writing, Editorial, and Other Assistance. Editorial assistance in the preparation of this article was provided by Dr. Yuanjiao Tang, Department of Ultrasound in West China Hospital of Sichuan University. Ultrasound examinations were provided by Dr. Yuanjiao Tang, Dr. Ling Zhong and Dr. Lingyan Zhang who are from the Department of Ultrasound in West China Hospital of Sichuan University.

Authorship. All named authors meet the International Committee of Medical Journal Editors (ICMJE) criteria for authorship for this article, take responsibility for the integrity of the work as a whole, and have given their approval for this version to be published.

Author Contributions. All authors contributed to the study conception and design. Material preparation, data collection and analysis were performed by YW, LZ and YC. All authors contributed to drafting and critical revision of the manuscript. All authors reviewed and approved the final version of the manuscript.

Disclosures. Yiyi Wang, Lingyan Zhang, Min Yang, Yanze Cao, Mingxin Zheng, Yuanxia $\mathrm{Gu}$, Hongxiang $\mathrm{Hu}$, Hui Chen, Min Zhang, Jingyi Li, Li Qiu, Wei Li have nothing to disclose.

Compliance with Ethics Guidelines. The study was approved by the ethics committee of West China Hospital, Sichuan University (approval number ChiCTR-DCD-15006851) and performed in accordance with the Helsinki Declaration of 1964, and its later amendments. Each participating patient signed an informed consent form. No identifying information of participants was included in the manuscript.

Data Availability. The datasets generated during and/or analyzed during the current study are available from the corresponding author on reasonable request.

Open Access. This article is licensed under a Creative Commons Attribution-NonCommercial 4.0 International License, which permits any non-commercial use, sharing, adaptation, distribution and reproduction in any medium or format, as long as you give appropriate credit to the original author(s) and the source, provide a link to the Creative Commons licence, and indicate if changes were made. The images or other third party material in this article are included in the article's Creative Commons licence, unless indicated otherwise in a credit line to the material. If material is not included in the article's Creative Commons licence and your intended use is not permitted by statutory regulation or exceeds the permitted use, you will need to obtain permission directly from the copyright holder. To view a copy of this licence, visit http://creativecommons.org/licenses/by$\mathrm{nc} / 4.0 /$. 


\section{REFERENCES}

1. Ogdie A, Weiss P. The epidemiology of psoriatic arthritis. Rheum Dis Clin N Am. 2015;41(4):545-68.

2. Ritchlin CT, Colbert RA, Gladman DD. Psoriatic arthritis. N Engl J Med. 2017;376(10):957-70.

3. Augustin M, Vietri J, Tian H, Gilloteau I. Incremental burden of cardiovascular comorbidity and psoriatic arthritis among adults with moderate-tosevere psoriasis in five European countries. J Eur Acad Dermatol Venereol. 2017;31(8):1316-23.

4. Taylor W, Gladman D, Helliwell P. Classification criteria for psoriatic arthritis: development of new criteria from a large international study. Arthritis Rheum. 2006;54(8):2665-73.

5. Scher JU, Ogdie A, Merola JF, Ritchlin C. Preventing psoriatic arthritis: focusing on patients with psoriasis at increased risk of transition. Nat Rev Rheumatol. 2019;15(3):153-66.

6. Savage L, Tinazzi I, Zabotti A, Laws PM, Wittmann M, McGonagle D. Defining pre-clinical psoriatic arthritis in an integrated dermato-rheumatology environment. J Clin Med. 2020;9(10):3262.

7. Weiner SM, Jurenz S, Uhl M, et al. Ultrasonography in the assessment of peripheral joint involvement in psoriatic arthritis: a comparison with radiography, MRI and scintigraphy. Clin Rheumatol. 2008;27(8):983-9.

8. Wiell C, Szkudlarek M, Hasselquist M, et al. Ultrasonography, magnetic resonance imaging, radiography, and clinical assessment of inflammatory and destructive changes in fingers and toes of patients with psoriatic arthritis. Arthritis Res Ther. 2007;9(6):R119.

9. Zabotti A, Bandinelli F, Batticciotto A, et al. Musculoskeletal ultrasonography for psoriatic arthritis and psoriasis patients: a systematic literature review. Rheumatology (Oxford). 2017;56(9): 1518-32.

10. Crespo-Rodriguez AM, Sanz Sanz J, Freites D, Rosales Z, Abasolo L, Arrazola J. Role of diagnostic imaging in psoriatic arthritis: how, when, and why. Insights Imaging. 2021;12(1):121.

11. Aydin SZ, Mathew AJ, Koppikar S, Eder L, Ostergaard $M$. Imaging in the diagnosis and management of peripheral psoriatic arthritis. Best Pract Res Clin Rheumatol. 2020;34(6):101594.

12. Gisondi P, Tinazzi I, El-Dalati G, et al. Lower limb enthesopathy in patients with psoriasis without clinical signs of arthropathy: a hospital-based casecontrol study. Ann Rheum Dis. 2008;67(1):26-30.

13. Simon D, Faustini F, Kleyer A, et al. Analysis of periarticular bone changes in patients with cutaneous psoriasis without associated psoriatic arthritis. Ann Rheum Dis. 2016;75(4):660-6.

14. Dubash SR, De Marco G, Wakefield RJ, Tan AL, McGonagle D, Marzo-Ortega H. Ultrasound imaging in psoriatic arthritis: what have we learnt in the last five years? Front Med (Lausanne). 2020;7:487.

15. Zabotti A, McGonagle DG, Giovannini I, et al. Transition phase towards psoriatic arthritis: clinical and ultrasonographic characterisation of psoriatic arthralgia. RMD Open. 2019;5(2):e001067.

16. Faustini F, Simon D, Oliveira I, et al. Subclinical joint inflammation in patients with psoriasis without concomitant psoriatic arthritis: a cross-sectional and longitudinal analysis. Ann Rheum Dis. 2016;75(12):2068-74.

17. Wang Y, Ding L, Chen J, et al. Risk factors for progression from subclinical to clinical phase of psoriatic arthritis: a case-control study. Rheumatol Ther. 2021;8(1):585-97.

18. (American College of Radiology (ACR), Society for Pediatric Radiology (SPR), Society of Radiologists in Ultrasound (SRU). AIUM practice guideline for the performance of a musculoskeletal ultrasound examination. J Ultrasound Med. 2012;31(9): 1473-1488.

19. Scheel AK, Hermann KG, Kahler E, et al. A novel ultrasonographic synovitis scoring system suitable for analyzing finger joint inflammation in rheumatoid arthritis. Arthritis Rheum. 2005;52(3): 733-43.

20. Szkudlarek M, Court-Payen M, Jacobsen S, Klarlund M, Thomsen HS, Ostergaard M. Interobserver agreement in ultrasonography of the finger and toe joints in rheumatoid arthritis. Arthritis Rheum. 2003;48(4):955-62.

21. Gottlieb AB, Bakewell C, Merola JF. Musculoskeletal imaging for dermatologists: techniques in the diagnosis and management of psoriatic arthritis. Dermatol Ther (Heidelb). 2021;11(4):1199-1216.

22. Zabotti A, Tinazzi I, Aydin SZ, McGonagle D. From psoriasis to psoriatic arthritis: insights from imaging on the transition to psoriatic arthritis and implications for arthritis prevention. Curr Rheumatol Rep. 2020;22(6):24.

23. Florescu A, Muşetescu AE, Florescu LM. The role of ultrasound in assessing hand joints and tendons in 
psoriatic arthritis. Curr Health Sci J. 2019;45(2): 198-203.

24. Elnady B, Shaarawy NK, El Dawoud NM, et al. Subclinical synovitis and enthesitis in psoriasis patients and controls by ultrasonography in Saudi Arabia; incidence of psoriatic arthritis during two years. Clin Rheumatol. 2019;38(6):1627-35.

25. Tinazzi I, McGonagle D, Biasi D, et al. Preliminary evidence that subclinical enthesopathy may predict psoriatic arthritis in patients with psoriasis. J Rheumatol. 2011;38(12):2691-2.

26. Gutierrez M, Filippucci E, De Angelis R, et al. Subclinical entheseal involvement in patients with psoriasis: an ultrasound study. Semin Arthritis Rheum. 2011;40(5):407-12.

27. Miedany Y, El Gaafary M, El Youssef S, Ahmed I, Nasr A. Tailored approach to early psoriatic arthritis patients: clinical and ultrasonographic predictors for structural joint damage. Clin Rheumatol. 2015;34(2):307-13.

28. Tang Y, Cheng S, Yang Y, et al. Ultrasound assessment in psoriatic arthritis (PsA) and psoriasis vulgaris (non-PsA): which sites are most commonly involved and what features are more important in PsA? Quant Imaging Med Surg. 2020;10(1):86-95.

29. Zabotti A, Piga M, Canzoni M, et al. Ultrasonography in psoriatic arthritis: which sites should we scan? Ann Rheum Dis. 2018;77(10):1537-8.

30. Naredo E, Moller I, de Miguel E, et al. High prevalence of ultrasonographic synovitis and enthesopathy in patients with psoriasis without psoriatic arthritis: a prospective case-control study. Rheumatology (Oxford). 2011;50(10):1838-48.

31. Tang Y, Yang Y, Xiang X, Wang L, Zhang L, Qiu L. Power Doppler ultrasound evaluation of peripheral joint, entheses, tendon, and bursa abnormalities in psoriatic patients: a clinical study. J Rheumatol. 2018;45(6):811-17.

32. Moshrif A, Mosallam A, Mohamed EE, Gouda W, Doma M. Subclinical enthesopathy in patients with psoriasis and its association with other disease parameters: a power Doppler ultrasonographic study. Eur J Rheumatol. 2017;4(1):24-28.

33. Zuliani F, Zabotti A, Errichetti E, et al. Ultrasonographic detection of subclinical enthesitis and synovitis a possible stratification of psoriatic patients without clinical musculoskeletal involvement. Clin Exp Rheumatol. 2019;37(4):593-9.

34. Wilson FC, Icen M, Crowson CS, McEvoy MT, Gabriel SE, Kremers HM. Incidence and clinical predictors of psoriatic arthritis in patients with psoriasis: a population-based study. Arthritis Rheum. 2009;61(2):233-9.

35. Soltani-Arabshahi R, Wong B, Feng BJ, Goldgar DE, Duffin KC, Krueger GG. Obesity in early adulthood as a risk factor for psoriatic arthritis. Arch Dermatol. 2010;146(7):721-6.

36. Eder L, Haddad A, Rosen CF, et al. The incidence and risk factors for psoriatic arthritis in patients with psoriasis: a prospective cohort study. Arthritis Rheumatol. 2016;68(4):915-23.

37. Solmaz D, Bakirci S, Al Onazi A, Al Osaimi N, Fahim S, Aydin SZ. Musculoskeletal ultrasound can improve referrals from dermatology to rheumatology for patients with psoriasis. Br J Dermatol. 2020;182(3):804-6.

38. Kampylafka E, Tascilar K, Lerchen V, et al. Secukinumab leads to shifts from stage-based towards response-based disease clusters-comparative data from very early and established psoriatic arthritis. Arthritis Res Ther. 2020;22(1):207. 